\title{
Implementación (parcial) en Ecuador de principios de la Ley Modelo CNUDMI, sobre arbitraje comercial. Retrospectiva histórica y necesidades
}

\author{
Eduardo Carmigniani* \\ Carla Cepeda** \\ Recibido/Received: 05/09/2017 \\ Aceptado/Accepted: 08/09/2017
}

\begin{abstract}
SUMARIO: 1. Introducción. 2. La Ley Modelo de la CNUDMI sobre arbitraje comercial (Ley Modelo). 2.1 Origen de la Ley Modelo. 2.2 Importancia de la implementación de la Ley Modelo. 3. Implementación de la Ley Modelo en la Ley de Arbitraje y Mediación del Ecuador (LAM). 3.1 Origen de la LAM. 3.2 Principios de la Ley Modelo que recoge la LAM. 3.2.1 Autonomía de la voluntad de las partes. 3.2.2 Separabilidad del convenio arbitral. 3.2.3 Kompetenz-kompetenz. 3.2.4 Autonomía del proceso arbitral. 3.2.5 Exclusión de intervención judicial. 3.2.6 Exclusión de intervención judicial. 4. Evaluación de la LAM. 4.1 Vacíos de la LAM. 4.1.1 Motivos de recusación de árbitros. 4.1.2 Práctica de la prueba. 4.1.3 Proceso de nulidad de laudos. 4.2 Desafíos que enfrenta el arbitraje comercial ecuatoriano. 5. Conclusión; modernización del arbitraje comercial ecuatoriano.
\end{abstract}

* Socio de Carmigniani Pérez Abogados y Presidente del Instituto Ecuatoriano de Arbitraje. Abogado por la Universidad Católica Santiago de Guayaquil. ecarmi@cplaw.ec.

** Asociada de Carmigniani Pérez Abogados. Abogada por la Universidad San Francisco de Quito. ccepeda@.cplaw.ec.

E. Carmigniani y C. Cepeda, "Implementación (parcial) en Ecuador de principios de la Ley Modelo CNUDMI, sobre arbitraje comercial. Retrospectiva histórica y necesidades", Revista Ecuatoriana de Arbitraje, No. 8, 2016. 
Implementación (parcial) en Ecuador de principios de la Ley Modelo CNUDMI, sobre arbitraje comercial. Retrospectiva histórica y necesidades

PALABRAS CLAVE: Ley Modelo CNUDMI, autonomía de la voluntad, modernización del arbitraje, flexibilidad, normas procesales, exclusión de prácticas judiciales.

KEYWORDS: UNCITRAL Model Law, party autonomy, modernization of arbitration, flexibility, rules of procedure, exclusion of judicial practices.

RESUMEN: El presente estudio pretende hacer un análisis sobre la implementación (parcial), en el Ecuador, de ciertos principios de la Ley Modelo, y la evolución del arbitraje comercial, igualmente en el Ecuador, a partir de la entrada en vigencia de la LAM. En primer lugar, se aborda el origen, los objetivos y la relevancia de la adopción de la Ley Modelo (respecto de cualquier jurisdicción). En segundo lugar, se sintetiza la historia legislativa del Ecuador en materia de arbitraje, la implementación (parcial) de la Ley Modelo en la LAM y los vacíos que nuestra legislación interna aún presenta. En tercer lugar, se presenta una evaluación de la legislación ecuatoriana en materia de arbitraje comercial, su aporte y beneficios, así como también los retos que actualmente enfrenta. Finalmente, se plantea soluciones a los actuales desafíos a los que se enfrenta el arbitraje comercial en Ecuador, tras veinte años de que entró en vigencia la LAM.

ABSTRACT: This article analyzes the (partial) implementation of the UNCITRAL Model Law and its principles in the Ecuadorian legal order. It also explains the evolution of the commercial arbitration in Ecuador since the enactment of the Arbitration and Mediation Law. First, the article examines the origin, objectives and relevance of the adoption of the UNCITRAL Model Law in all jurisdictions. Secondly, a study is performed on the historical arbitration legislation, the partial implementation of the Model Law in our legal order and the internal legislative gaps it still has to overcome. Third, the paper introduces an evaluation of the Ecuadorian law on arbitration, its contribution and benefit to the legal community, identifying its current challenges. Finally, the article in- 
troduces possible plans of action to address those challenges, after 20 years since the Ecuadorian Arbitration and Mediation Law was enacted.

\section{INTRODUCCIÓN}

La Comisión de las Naciones Unidas para el Derecho Mercantil Internacional (CNUDMI) creó uno de los instrumentos más importante en materia de arbitraje comercial, la Ley Modelo. Este esquema normativo establece directrices de absoluta relevancia para solventar los problemas que se evidencian en las legislaciones internas de distintos países para la implementación del arbitraje comercial y su efectivo funcionamiento. La Ley Modelo pretende difundir, unificar y consolidar el funcionamiento del arbitraje comercial en los distintos países, mediante su adopción como normativa interna.

La LAM tomó de la Ley Modelo una serie de principios y lineamientos fundamentales en materia de arbitraje. La LAM se ha convertido en una importante herramienta para la resolución de conflictos en el Ecuador mediante arbitraje. Sin embargo, a pesar de los notorios avances alcanzados durante sus veinte años de vigencia, es de toda evidencia la actual necesidad de dar nuevos pasos dirigidos a la modernización del sistema en nuestro país.

Este trabajo presenta: (i) un detalle sobre los aspectos más relevantes de la Ley Modelo y la importancia de su implementación; (ii) la evolución del arbitraje en el Ecuador y la implementación (parcial) de los principios de la Ley Modelo en la LAM; (iii) una evaluación de la LAM desde su puesta en vigencia hasta la actualidad; (iv) las necesidades que el arbitraje enfrenta actualmente en el Ecuador; (v) propuestas para la modernización del arbitraje en Ecuador. 
Implementación (parcial) en Ecuador de principios de la Ley Modelo CNUDMI, sobre arbitraje comercial. Retrospectiva histórica y necesidades

\section{LA Ley Modelo}

La Ley Modelo es uno de los instrumentos más importantes con los que cuenta el arbitraje comercial a nivel internacional. $\mathrm{Su}$ modelo de legislación interna ha sido adoptado íntegramente en varias jurisdicciones y en otras ha servido como referencia legislativa y judicial ${ }^{1}$. Su objetivo principal es unificar las prácticas arbitrales a nivel internacional y propender al funcionamiento uniforme de este sistema.

\subsection{Origen de la Ley Modelo}

La Ley Modelo nació como una propuesta para solventar los problemas identificados en la práctica de arbitraje comercial internacional y así reducir los obstáculos que enfrentaba el sistema en ese entonces ${ }^{2}$. La elaboración de los primeros trabajos de investigación y borradores de la Ley Modelo se realizaron con la contribución de organizaciones internacionales interesadas en la materia, particularmente lideradas por el Comité Consultivo Jurídico Asiático-Africano y el Consejo Internacional de Arbitraje Comercial $^{3}$. Dentro de las primeras conclusiones a las que llegó la comisión redactora de la Ley Modelo y que enmarcarían los límites de su aplicación fueron: (i) que su ámbito de aplicación estaría restringido al arbitraje comercial y (ii) que las disposiciones de la Ley Modelo debían ser uniformes con la Convención de Nueva York de $1958^{4}$.

Delimitado el alcance de la Ley Modelo, los principales retos que el arbitraje comercial internacional enfrentaba en ese momento eran fundamentalmente restricciones a: (i) la autonomía

1. G. BoRn. International Commercial Arbitration, Kluwer Law, 2014, p. 134.

2. Ibídem.

3. Report of the Secretary-General on Possible Features of a Model Law on International Commercial Arbitration, U.N. Doc. A/CN.9/207, XII, Year Book UNCITRAL 1981, p. 76.

4. Ídem, p. 76. "One of UNCITRAL's aims through the Model Law was to reduce de divergences which might result from each State's interpretations of its obligations under de New York Convention". Traducción libre: Uno de los objetivos fundamentales de la Ley Modelo era reducir las divergencias que resultaran de la interpretación que cada Estado realizara sobre las obligaciones que derivan de la Convención de Nueva York. 
de la voluntad de las partes para someter disputas a arbitraje y convenir las reglas de procedimiento que considerasen más convenientes; (ii) la forma de designación de árbitros; (iii) la potestad de los árbitros para juzgar sobre su competencia o la manera de conducir el proceso arbitral; (iv) la selección de la legislación aplicable. Adicionalmente, se criticaba la intervención e indeseable intromisión de los órganos jurisdiccionales en el proceso arbitral ${ }^{5}$.

El 21 de junio de 1985, la CNUDMI aprobó la Ley Modelo sobre Arbitraje Comercial Internacional ${ }^{6}$. Meses después, la Asamblea General de Naciones Unidas recomendó “que todos los Estados examinen debidamente la (Ley Modelo), teniendo en cuenta la conveniencia de la uniformidad del derecho procesal arbitral y las necesidades específicas de la práctica del arbitraje comercial internacional" ${ }^{\prime 7}$.

La Ley Modelo contiene 36 artículos que abarcan una serie de problemáticas que se discuten en los procedimientos arbitrales a nivel nacional e internacional ${ }^{8}$. Entre las disposiciones más destacadas se encuentran (i) la validez y eficacia del convenio arbitral (artículos 7 a 9); (ii) el modo de designación, excusa y recusación de árbitros (artículos 10-15); la jurisdicción de los árbitros y aplicación de medidas provisionales (artículos 16-17); conducción general del proceso arbitral (artículos 18-26); práctica de la prueba (artículo 27); aplicación de la ley de fondo (artículo 28); emisión del laudo arbitral y acciones en contra del laudo (artículos 29-34); reconocimiento y ejecución de laudos internacionales (artículos 35-36).

En el año 2006, la CNUDMI realizó una primera -y hasta el momento única- enmienda a la Ley Modelo, tendiente a incluir temas antes no considerados; a aclarar ciertas disposiciones y a complementar otras. Así, la enmienda implementa normas (i)

5. Report of the Secretary-General on Possible Features of a Model Law on International Commercial Arbitration, N. 3, p. 77.

6. Nota explicativa de la secretaría de la CNUDMI acerca de la Ley Modelo sobre Arbitraje Comercial Internacional de 1985, en su versión enmendada en 2006, p. 25.

7. Asamblea General Naciones Unidas, Resolución 40/72, 11/12/1985.

8. Ley Modelo CNUDMI sobre Arbitraje Comercial Internacional de 1985 con las enmiendas aprobadas en 2006 (Ley Modelo CNUDMI) (2008), Art. 36. 
Implementación (parcial) en Ecuador de principios de la Ley Modelo CNUDMI, sobre arbitraje comercial. Retrospectiva histórica y necesidades

sobre la aplicación de principios generales de interpretación; (ii) aclaraciones sobre el requisito de que el convenio arbitral conste por escrito; (iii) sobre la facultad de las partes para solicitar medidas provisionales al Tribunal Arbitral o a las cortes nacionales y (iv) sobre el proceso de reconocimiento y ejecución de laudos arbitrales.

En definitiva, la Ley Modelo fue diseñada para que sus disposiciones sean implementadas en las legislaciones internas con el objetivo de armonizar el manejo del arbitraje comercial internacional y asegurar su reconocimiento y efectivo funcionamiento 9 . La CNUDMI ha realizado notables esfuerzos para actualizar e implementar directrices legislativas sobre el manejo del sistema arbitral. Esto se debe -sin lugar a dudas- a la importancia que el arbitraje representa para afianzar las relaciones comerciales internacionales.

\subsection{Importancia de la implementación de la Ley Modelo}

Como se mencionó, la Ley Modelo es una de las herramientas más importantes que discute y solventa las problemáticas que suelen presentarse en el arbitraje comercial. Adicionalmente, este cuerpo normativo ha sido estudiado por las instituciones arbitrales y expertos más destacados y prestigiosos en la materia. Por consiguiente, es indiscutible la calidad del documento, la importancia de su estudio y la necesidad de su implementación. Todo Estado que pretenda contar con un moderno sistema de arbitraje comercial, necesariamente debe adoptar en su normativa interna -al menos- los lineamientos y principios que enmarcan la Ley Modelo.

La Ley Modelo ha sido adoptada, por ejemplo, por Alemania, Australia, Canadá, Costa Rica, República Dominicana, Hong Kong, India, México, Perú, Singapur ${ }^{10}$. En el caso de Ecuador,

9. Report of the Secretary-General on Possible Features of a Model Law on International Commercial Arbitration, N. 3, pp. 74-75; E. Gaillard y J. SAVAGE (Eds.), Fouchard Gaillard Goldman on International Commercial Arbitration, 1999, p. 70; G. BoRN, N. 1, p. 135.

10. Ídem, p. 138. 
como se observará más adelante, la LAM incorporó algunos lineamientos de la Ley Modelo.

Los motivos por los que la Ley Modelo ha obtenido una considerable acogida son innumerables. La Ley Modelo se creó como el instrumento tendiente a armonizar y a mejorar la perspectiva y flexibilidad que se requiere de los Estados al momento de elaborar su normativa interna en materia de arbitraje ${ }^{11}$. Entre las ventajas más destacadas que representa la adopción de la Ley Modelo se encuentran: (i) que brinda accesibilidad a los operadores del sistema para acceder a otros países; (ii) que garantiza la uniformidad internacional en materia de arbitraje y (iii) que representa una estructura ya probada y funcionalmente eficiente.

En este sentido, sobre la importancia de la implementación de la Ley Modelo, el ministro de justicia de Alemania manifestó:

[I]f we want to reach the goal that Germany will be selected more frequently as the seat of international arbitrations in the future, we have to provide foreign parties with a law that, by its outer appearance and by its contents, is in line with the framework of the Model Law that is so familiar all over the world ${ }^{12}$.

Por otra parte, la Comisión de Hong Kong encargada de realizar una reforma a su legislación interna de arbitraje comercial expresó "the Model Law [...] has the advantage of making [Hong Kong] law internationally recognizable and accesible"13.

A pesar de los importantes beneficios que la adopción de la Ley Modelo representa, varios países como Francia, Suiza, Inglaterra, Holanda, entre otros, han decidido no adoptar íntegramente el texto de la Ley Modelo en su normativa interna, sino utilizarla simplemente como referencia. Esto se debe a que han

11. M. SECCOB, Shades of delocalisation-Diversity in the Adoption of the UNCITRAL Model Law in Australia, Hong Kong and Singapore, Journal of International Arbitration, Kluwer Law International, Vol. 17, 2000, p. 1.

12. Bundestags-Drucksache No. 13/5274, 12/07/1996, The New German Arbitration Law 140, 1998. Citado en G. Born, N. 1, p. 139.

13. Law Reform Commission of Hong Kong, Report on the Adoption of the UNCITRAL Model Law of Arbitration, 1987. Citado en G. BorN, N. 1, p. 139. 
Implementación (parcial) en Ecuador de principios de la Ley Modelo CNUDMI, sobre arbitraje comercial. Retrospectiva histórica y necesidades

considerado que la Ley Modelo podría resultar muy extensa y ciertas disposiciones podrían simplificarse o adaptarse a las necesidades internas de cada país ${ }^{14}$. Es claro que estos países siguen reconociendo el importante valor y aporte de los principios recogidos en la Ley Modelo para su legislación interna, simplemente no han adoptado íntegramente su texto.

\section{IMPlementación de LA Ley Modelo en LA LAM}

Como ya se dijo, es indiscutible la importancia de la Ley Modelo para el desarrollo del arbitraje comercial y para la adopción de las mejores prácticas internacionales. El caso del Ecuador es particular; si bien acogió en la LAM ciertos lineamientos de la Ley Modelo, aún existen muchos por desarrollarse y que requieren una revisión, tal y como se verá en las siguientes secciones.

\subsection{Origen de la LAM}

La primera ley especial que reguló el arbitraje en el Ecuador fue la Ley de Arbitraje Comercial ${ }^{15}$, de octubre de 1963. Antes de eso, el decimonónico Código de Procedimiento Civil ("CPC") regulaba, en su sección 33, el denominado "juicio por arbitraje", que recogía un primer intento de admitir la celebración de un convenio arbitral. "Se llama compromiso al contrato por el cual dos o más personas someten al juicio de arbitraje sus diferencias. El compromiso se hará constar en escritura pública o en documento privado reconocido por las partes $[\ldots]^{\prime \prime 16}$. Las disposiciones del Código de Procedimiento Civil respecto al sistema arbitral eran imprácticas y resultaban excesivamente formalistas. La Ley de Arbitraje Comercial reconoció al arbitraje como mecanismo idóneo para la resolución de controversias entre comerciantes y otorgaba a las cámaras de comercio la facultad de prestar sus ser-

14. E. Gaillard y J. Savage (Eds.), M. 9, p. 109.

15. Ley de Arbitraje Comercial (derogada), Decreto Supremo No. 735, 23/10/1963, RO N. 90, 28/10/1963.

16. Código de Procedimiento Civil ecuatoriano (derogado), RO Sup. No. 133, 7/02/1953. 
vicios para administrar estos procesos ${ }^{17}$. A pesar del significativo avance que representaba una ley especial en la materia, su utilización no fue significativa por "desconocimiento, falta de promoción, entre otras razones" ${ }^{\prime 18}$.

A mediados de la década de los 90 del siglo pasado, la ONG Corporación Latinoamericana para el Desarrollo promovió la redacción de un proyecto de ley que regulase el arbitraje comercial en el Ecuador, con la finalidad de incentivar su uso, hasta entonces prácticamente inexistente. Este esfuerzo culminó con la promulgación de la LAM, en $1997^{19}$.

\subsection{Principios de la Ley Modelo que recoge la LAM}

En la LAM se adoptó varios principios fundamentales inspirados en la Ley Modelo. Entre ellos, se destacan:

\subsubsection{Autonomía de la voluntad de las partes}

No es un tema controvertido que el principio rector y fundamental en materia de arbitraje comercial es el de autonomía de la voluntad de las partes. Bajo este principio, las partes tienen libertad para conferir a los árbitros potestades suficientes para dirimir su controversia y para establecer o delimitar las reglas bajo las cuales se regirá el procedimiento arbitral ${ }^{20}$.

El principio de autonomía de la voluntad es considerado como la base fundamental de la Ley Modelo ${ }^{21}$, y está recogido expresamente en su artículo 7: "[e]l "acuerdo de arbitraje" es un

17. A. Galindo, Origen y Desarrollo de la Solución Alternativa de Conflictos en Ecuador, Iuris Dictio, año II, No. 4, 2011, p. 123.

18. Ibídem.

19. Ley de Arbitraje y Mediación ecuatoriana (LAM), RO. No. 145, 04/09/1997.

20. E. Gaillard \& J. SaVage (Eds.), N. 9, p. 3; A. REDFERn et al., Law and Practice of International Commercial Arbitration, London, Tomson \& Maxwell, 2004, p. 265.

21. Report of the Secretary-General on Possible Features of a Model Law on International Commercial Arbitration, N. 3: "Probably the most important principle on which the Model Law should be based is the freedom of the parties in order to facilitate the proper functioning of international commercial arbitrations according to their expectation", p. 78. 
Implementación (parcial) en Ecuador de principios de la Ley Modelo CNUDMI, sobre arbitraje comercial. Retrospectiva histórica y necesidades

acuerdo por el que las partes deciden someter a arbitraje todas las controversias o ciertas controversias que hayan surgido o puedan surgir entre ellas $[\ldots]^{22}$. En concordancia con la Ley Modelo, el artículo 5 de la LAM prescribe que el convenio arbitral es "el acuerdo escrito en virtud del cual las partes deciden someter a arbitraje todas o ciertas controversias que hayan surgido entre ellas [...]". Estas disposiciones determinan con claridad la libertad que existe para que las partes acuerden someter sus disputas a arbitraje y de hecho enfatiza que la legitimidad de este sistema procesal surge precisamente de ese acuerdo libre y voluntario. En consecuencia, la naturaleza jurídica del arbitraje es en esencia negocial.

Otra disposición que regula con absoluta relevancia el principio de autonomía de la voluntad es el artículo 19(1) de la Ley Modelo, el cual prevé que "[c]on sujeción a las disposiciones de la presente Ley, las partes tendrán libertad para convenir el procedimiento a que se haya de ajustar el tribunal arbitral en sus actuaciones" ${ }^{23}$ (énfasis añadido). Esta disposición, a su vez, se encuentra en armonía con la Convención de Nueva York, la cual en su artículo $\mathrm{V}(1)(\mathrm{d})$ prescribe que se podrá denegar el reconocimiento y la ejecución de un laudo extranjero si "la constitución del tribunal arbitral o el procedimiento arbitral no se han ajustado al acuerdo celebrado entre las partes $[\ldots]^{24 \prime \prime}$ (énfasis añadido).

A su vez, la LAM recoge una disposición similar en la que otorga a las partes la libertad de acordar el procedimiento que regirá el arbitraje. Así, el artículo 38 prescribe que "[e]l arbitraje se sujetará a las normas de procedimiento señaladas en esta Ley, al procedimiento establecido en los centros de arbitraje, al determinado en el convenio arbitral o al que las partes escojan [...]" (énfasis añadido). Adicionalmente, existen otras disposiciones en las que se evidencia la aplicación del principio de autonomía de la voluntad de las partes en la LAM. Por ejemplo, los contratantes se encuentran facultados para decidir (i) si el arbitraje será adminis-

22. Ley Modelo CNUDMI, N. 8, Art. 7, opción II.

23. Ley Modelo CNUDMI, N. 8.

24. Convención sobre el Reconocimiento y la Ejecución de las Sentencias Arbitrales Extranjeras, Nueva York, (1958). 
trado o ad-hoc ${ }^{25}$; (ii) la forma de selección de árbitros ${ }^{26}$; (iii) si se resolverá en derecho o en equidad ${ }^{27}$; (iv) el lugar del arbitraje ${ }^{28}$; (v) la naturaleza del conflicto que someterán a arbitraje ${ }^{29}$ y en general -como se ha mencionado- (vi) las reglas que regirán el proceso $^{30}$.

Por lo expuesto, es claro que la LAM contiene normas expresas en las que se reconoce la aplicación del principio de autonomía de la voluntad como medular en el sistema arbitral. Su incorporación al sistema ecuatoriano constituye un avance y permite que la regulación del arbitraje comercial se acople a las mejores prácticas internacionales.

\subsubsection{Separabilidad del convenio arbitral}

Otro principio esencial del arbitraje, que se encuentra regulado en la LAM y que ha sido inspirado por la Ley Modelo, es el de separabilidad o autonomía del convenio arbitral. Este principio representa una ficción jurídica respecto de la relación que existe entre el convenio arbitral y el contrato sustantivo objeto de la controversia ${ }^{31}$. Según este principio, el convenio arbitral es independiente del contrato principal. De esa forma, el primero no se ve necesariamente afectado por los vicios que podrían invalidar o afectar la eficacia del último, por el solo hecho de estar incorporados en el mismo documento ${ }^{32}$ (salvo que los vicios sean

25. LAM, N. 19, Art. 2 "El arbitraje es administrado cuando se desarrolla con sujeción a esta Ley y a las normas y procedimientos expedidos por un centro de arbitraje, y es independiente cuando se realiza conforme a lo que las partes pacten, con arreglo a esta Ley".

26. LAM, N. 19, Art. 16 "De no existir acuerdo total en la audiencia de mediación, el director del centro de arbitraje enviará a las partes la lista de árbitros, para que de común acuerdo designen en el término de tres días los árbitros principales y el alterno que deban integrar el tribunal".

27. LAM, N. 19, Art. 3 "Las partes indicarán si los árbitros deben decidir en equidad o en derecho, a falta de convenio, el fallo será en equidad".

28. LAM, N. 19, Art. 35 "De no constar en el convenio, las partes podrán determinar libremente el lugar del arbitraje [...]".

29. LAM, N. 19, Art. 5 "El convenio arbitral es el acuerdo escrito en virtud del cual las partes deciden someter a arbitraje todas las controversias o ciertas controversias que hayan surgido o puedan surgir entre ellas respecto de una determinada relación jurídica, contractual o no contractual".

30. LAM, N. 19, Art. 38.

31. E. Onyema. International Commercial Arbitration and the Arbitrator's Contract. Oxon: Routledge Research in International Commercial Law, p. 20; Nota explicativa de la secretaría de la CNUDMI..., N. 6, p. 33.

32. Ibídem. 
Implementación (parcial) en Ecuador de principios de la Ley Modelo CNUDMI, sobre arbitraje comercial. Retrospectiva histórica y necesidades

comunes, v. gr., si ambos están afectados por el mismo vicio del consentimiento). Adicionalmente, otra de las principales consecuencias de la aplicación de este principio es que genera la posibilidad de convenir diferentes legislaciones o normativa que por un lado gobiernen al contrato sustantivo y por otro lado al convenio arbitral ${ }^{33}$.

La Ley Modelo recoge el principio de autonomía del convenio arbitral en su artículo 16(1), el cual prescribe:

[...] una cláusula compromisoria que forme parte de un contrato se considerará como un acuerdo independiente de las demás estipulaciones del contrato. La decisión del tribunal arbitral de que el contrato es nulo no entrañará ipso jure la nulidad de la cláusula compromisoria".

En igual sentido, la LAM también recoge el principio de autonomía del convenio arbitral. Así, su artículo 5 categóricamente dispone "la nulidad de un contrato no afectará la vigencia del convenio arbitral".

\subsubsection{Kompetenz-Kompetenz}

El principio Kompetenz-Kompetenz consiste en que los árbitros tienen la potestad para decidir sobre su propia competencia para conocer la controversia sometida a arbitraje, incluso aquellas relativas a la inexistencia e invalidez del convenio arbitral, sin necesidad de acudir a la justicia ordinaria ${ }^{34}$. Esta doctrina ha sido ampliamente estudiada e incluso criticada por considerar que los árbitros podrían encontrarse estimulados o influenciados para fallar a favor de su competencia para no perder la oportunidad de sustanciar un arbitraje ${ }^{35}$. Sin embargo, este principio ha sido mayoritariamente aceptado, considerando que debe primar la voluntad de las partes de someter todas las controversias a arbitraje

\footnotetext{
33. Ibídem.

34. Ibídem.

35. M. Moses, The Principles and Practice of International Commercial Arbitration, Cambridge University Press, 2008, p. 88.
} 
-incluso las concernientes a la jurisdicción y competencia del tribunal arbitral- sobre la posible tendencia de los árbitros a favorecer su competencia para conocer la disputa ${ }^{36}$.

En la Ley Modelo, es posible observar la inclusión del principio Kompetenz-Kompetenz en el artículo 16(1), el cual dispone que "El tribunal arbitral estará facultado para decidir acerca de su propia competencia, incluso sobre las excepciones relativas a la existencia o a la validez del acuerdo de arbitraje [...]". Por su parte, el artículo 22 de la LAM establece que "[u]na vez constituido el tribunal, se fijará día y hora para la audiencia de sustanciación en la que se posesionará el secretario designado, se leerá el documento que contenga el convenio arbitral y el tribunal resolverá sobre su propia competencia" (énfasis añadido).

En torno a este principio ha surgido una discusión que reviste particular importancia comparativa entre la Ley Modelo y la LAM. En el foro arbitral existen cuestionamientos respecto a si las decisiones sobre competencia adoptadas por los árbitros deberían ser definitivas o si por el contrario podrían ser revisadas por otra autoridad. De acuerdo a la Ley Modelo, un Tribunal Arbitral puede resolver sobre su propia competencia como una cuestión preliminar o en un laudo final ${ }^{37}$. En el primer caso, en el que los árbitros aborden la decisión de competencia como preliminar, las partes podrán solicitar en 30 días una revisión de esta decisión a una autoridad competente escogida por ellas, quien finalmente podrá juzgar si la decisión de competencia del tribunal arbitral fue apropiada ${ }^{38}$.

Por otra parte, en nuestro ordenamiento jurídico, la decisión del Tribunal Arbitral sobre competencia en principio debe adoptarse como una cuestión preliminar durante la audiencia de sustanciación, salvo que para decidirla haya que pronunciarse inextricablemente sobre el fondo de la controversia, en cuyo caso hay reglamentos de arbitraje, como el del Centro de Arbitraje de

\footnotetext{
36. Ibídem.

37. Ley Modelo CNUDMI, N. 8, Art. 16 (3).

38. M. Moses, N. 35, p. 90
} 
Implementación (parcial) en Ecuador de principios de la Ley Modelo CNUDMI, sobre arbitraje comercial. Retrospectiva histórica y necesidades

la Cámara de Comercio de Guayaquil, que permite al tribunal arbitral reservar la decisión sobre competencia para expedirla en el laudo. En el primer caso, la decisión sobre competencia, adoptada en la audiencia de sustanciación, pudiera ser impugnada, por vía de inconstitucionalidad, por violación de la garantía del debido proceso al juez competente, ante la Corte Constitucional mediante la acción extraordinaria de protección regulada en el artículo 94 de la Constitución. En el segundo caso, hay discusión no resuelta, y más bien inundada de decisiones contradictorias, en torno a si la declaración de competencia efectuada en laudo puede ser impugnada por vía directa, mediante la misma acción extraordinaria de protección, o si previamente debe plantearse una acción de nulidad de laudo, regulada en el artículo 31 de la LAM.

\subsubsection{Autonomía del proceso arbitral}

El principio de autonomía del proceso arbitral, que se encuentra esencialmente relacionado con el principio de autonomía de la voluntad, es quizá uno de los principios más importantes en materia de arbitraje, pues es precisamente el que lo aleja de la jurisdicción ordinaria y lo cataloga como un mecanismo procesal alternativo de resolución de disputas. En este sentido, la Secretaría General de Naciones Unidas en su reporte sobre la Ley Modelo manifestó: "probably the most important principles on which the Model Law should be based is the freedom of the parties in order to facilitate the proper functioning of the international arbitrations according to their expectations" 39 .

Este principio es crucial para el desarrollo del arbitraje como un mecanismo alternativo de resolución de disputas, ya que busca excluir los ritualismos y formalidades de los procesos ante la justicia estatal, permitiendo que las partes puedan escoger normas más eficientes y acorde a sus intereses. La lógica detrás de este principio es que el arbitraje -a diferencia del proceso judi-

39. Report of the Secretary-General on Possible Features of a Model Law on International Commercial Arbitration, N. 3, p. 75. 
cial- pueda alejarse de las prácticas ordinarias que en la mayoría de casos entorpecen la resolución del conflicto.

La Ley Modelo reconoce la importancia de este principio en varias de sus disposiciones. Particularmente, el artículo 19(1) prescribe que "[c]on sujeción a las disposiciones de la presente Ley, las partes tendrán libertad para convenir el procedimiento a que se haya de ajustar el tribunal arbitral en sus actuaciones". Adicionalmente, el artículo 24(1) de la Ley Modelo expresamente permite a las partes decidir sobre la forma de presentar su caso ante el Tribunal Arbitral y los temas relativos a la práctica de la prueba $^{40}$.

La LAM también reconoce el principio de autonomía del proceso arbitral. Así, el artículo 38 prescribe que:

[e]l arbitraje se sujetará a las normas de procedimiento señaladas en esta Ley, al procedimiento establecido en los centros de arbitraje, al determinado en el convenio arbitral o al que las partes escojan, sin perjuicio de las normas supletorias que sean aplicables (énfasis añadido).

El problema se presenta en los casos en los que las partes han sido silentes sobre las reglas que regirán el proceso arbitral, lo que ha llevado a sostener que deberían aplicarse directamente las normas procesales supletorias. Desde nuestra perspectiva esa postura es errada. La LAM ha reconocido el principio de autonomía de las partes para regular el proceso arbitral, inspirándose en la Ley Modelo, y esta prevé que en caso de que las partes no hayan pactado reglas específicas para el proceso, el tribunal arbitral tendrá la potestad para decidir sobre ellas.

40. Ley Modelo CNUDMI, N. 8, Art. 24 “Audiencias y actuaciones por escrito (1) Salvo acuerdo en contrario de las partes, el tribunal arbitral decidirá si han de celebrarse audiencias para la presentación de pruebas o para alegatos orales, o si las actuaciones se sustanciarán sobre la base de documentos y demás pruebas. No obstante, a menos que las partes hubiesen convenido que no se celebrarían audiencias, el tribunal arbitral celebrará dichas audiencias en la fase apropiada de las actuaciones, a petición de una de las partes". 
Implementación (parcial) en Ecuador de principios de la Ley Modelo CNUDMI, sobre arbitraje comercial. Retrospectiva histórica y necesidades

Así, el artículo 19(2) de la Ley Modelo prescribe

[a] falta de acuerdo, el tribunal arbitral podrá, con sujeción a lo dispuesto en la presente Ley, dirigir el arbitraje del modo que considere apropiado. Esta facultad conferida al tribunal arbitral incluye la determinación, admisibilidad, la pertinencia y el valor de las pruebas.

De acuerdo a la nota explicativa de la Ley Modelo la facultad discrecional supletoria del Tribunal Arbitral

[E]s igualmente importante pues permite que éste sustancie las actuaciones según las características especiales de cada caso, sin las limitaciones impuestas por la legislación interna que suele aplicarse, incluso la que rige la práctica de la prueba. Además proporciona la base para dar muestras de iniciativa a fin de solucionar toda cuestión procesal no contemplada en el acuerdo de arbitraje ni en la Ley Modelo ${ }^{41}$.

Esta potestad supletoria que se otorga al tribunal arbitral en la Ley Modelo, nace del encargo de confianza que las partes han otorgado a los árbitros para resolver su controversia de la forma más prolija posible.

En nuestro ordenamiento jurídico también se encuentra reconocida esta potestad del tribunal arbitral, y particularmente de su presidente. Así, el artículo 17 de la LAM faculta al presidente del tribunal arbitral para sustanciar y dirigir el proceso, por lo que para nada es ajena a la LAM la potestad del tribunal, o de su presidente, para determinar las normas de procedimiento que mejor convengan a la continuación del arbitraje, siempre y cuando las partes no convengan algo diferente.

En el Ecuador existe una corriente de pensamiento favorable a considerar que el arbitraje es un mecanismo que permite (i) a las partes diseñar su propio procedimiento de resolución de disputas; (ii) adoptar las mejores prácticas internacionales y (iii) alejarse de los ritualismos de los procesos judiciales. Sin embargo,

41. Nota explicativa de la secretaría de la CNUDMI ..., N. 6, pp. 35-36. 
en la práctica esto resulta relativamente más complejo que en la teoría. Generalmente, los empresarios y comerciantes no incluyen reglas de procedimiento en sus convenios arbitrales. Por otra parte, los reglamentos de los centros de arbitraje ecuatorianos, en su mayoría, son silentes sobre cómo sustanciar el arbitraje en los temas no regulados por la LAM y las partes. Esto ha creado un aparente vacío que -incorrectamente- ha sido llenado por algunos tribunales arbitrales con el abuso de normas supletorias ${ }^{42}$, contradiciendo, en esta forma, uno de los objetivos principales por los que las partes escogieron someterse a arbitraje, esto es excluir las prácticas ritualistas comunes en los procesos judiciales.

En definitiva, es claro que la LAM recoge el principio de autonomía del proceso arbitral adoptado de la Ley Modelo, pues expresamente permite a las partes convenir las reglas de procedimiento que consideren convenientes. Adicionalmente, la LAM faculta al presidente del tribunal arbitral a sustanciar y manejar el proceso de la manera más conveniente para el caso concreto. Sin embargo, el desconocimiento sobre el alcance de aplicación de este principio y los aparentes vacíos normativos sobre las reglas que rigen al proceso arbitral, han provocado que los operadores del sistema arbitral abusen de las normas supletorias a las que se refiere el artículo 37 de la LAM e intoxiquen al arbitraje con las normas ritualistas que rigen los procesos judiciales ordinarios.

\subsubsection{Exclusión de intervención judicial}

Uno de los principios trascendentales que inspira la Ley Modelo es la exclusión de la intervención judicial en el arbitraje. Como se mencionó en las secciones iniciales de este artículo, la CNUDMI identificó que la intervención de la justicia ordinaria en el arbitraje era uno de los principales problemas que enfrentaba el sistema Arbitral. En la actualidad existe una tendencia a limitar esta intervención, lo cual "se justifica porque las partes en

42. LAM, N. 19, Art. 37 "En todo lo que no esté previsto en esta Ley, se aplicarán supletoriamente las normas del Código Civil, Código de Procedimiento Civil o Código de Comercio y otras leyes conexas, siempre que se trate, de arbitraje en derecho". 
Implementación (parcial) en Ecuador de principios de la Ley Modelo CNUDMI, sobre arbitraje comercial. Retrospectiva histórica y necesidades

un acuerdo de arbitraje adoptan deliberadamente la decisión de excluir la competencia judicial y prefieren la conveniencia práctica y la irrevocabilidad del proceso arbitral" ${ }^{\prime 3}$.

En este sentido, la Ley Modelo restringe o delimita expresamente las situaciones en las que podría intervenir una autoridad judicial, situaciones que se pueden clasificar en dos grupos: el primero consiste en la intervención de autoridades públicas en temas relativos al nombramiento, recusación de árbitros, terminación del mandato de árbitros; a los temas relativos a revisión de competencia del Tribunal Arbitral -ya discutido-y a la potestad de anular el laudo arbitral ${ }^{44}$. Por otra parte, un segundo grupo se refiere a la intervención judicial de manera complementaria y auxiliar al arbitraje en temas relativos a la práctica de prueba, medidas cautelares y reconocimiento y ejecución de laudos ${ }^{45}$.

El Ecuador ha adoptado satisfactoriamente este principio, pues el artículo 7 de la LAM prescribe:

[e]l convenio arbitral, que obliga a las partes a acatar el laudo que se expida, impide someter el caso a la justicia ordinaria. Cuando las partes hayan convenido de mutuo acuerdo someter a arbitraje sus controversias, los jueces deberán inhibirse de conocer cualquier demanda que verse sobre las relaciones jurídicas que las hayan originado, salvo en los casos de excepción previstos en esta Ley. En caso de duda, el órgano judicial respectivo estará a favor de que las controversias sean resueltas mediante arbitraje. Toda resolución a este respecto deberá ser notificada a las partes en el término de dos días.

Adicionalmente, en la práctica este principio ha sido correctamente aceptado. Tanto los partícipes en el sistema arbitral como los jueces ordinarios reconocen que la existencia de un convenio arbitral prima facie excluye la intervención de las cortes estatales

43. Nota explicativa de la secretaría de la CNUDMI acerca de la Ley Modelo sobre Arbitraje Comercial Internacional de 1985, en su versión enmendada en 2006, pp. 29 -30.

44. Estos temas serán resueltos por la autoridad escogida por las partes, de acuerdo a lo previsto en el artículo 6 de la Ley Modelo.

45. Nota explicativa de la secretaría de la CNUDMI acerca de la Ley Modelo sobre Arbitraje Comercial Internacional de 1985, en su versión enmendada en 2006, pp. 29 -30. 
para conocer la controversia. Por otra parte, es aceptado que se requerirá el auxilio judicial para temas específicos conexos al arbitraje. Por ejemplo, para la ejecución de medidas cautelares, ejecución de laudos arbitrales y para conocer el proceso de nulidad de laudos arbitrales.

\section{Evaluación de la LAM}

Como ha quedado dicho, la LAM recoge varios de los principios más importantes que rigen el arbitraje comercial internacional, recogidos en la Ley Modelo. Nuestra legislación no incorpora como ley interna a la Ley Modelo. No obstante, ha logrado adaptar e implementar ciertas bases fundamentales.

La LAM, en sus veinte años de vigencia, ha permitido que el arbitraje se desarrolle e institucionalice en el Ecuador. Desde su promulgación los casos de arbitraje se han incrementado y se siguen incrementando considerablemente. Durante estos veinte años se han realizado en nuestro país varios miles de arbitrajes, en los distintos centros que los administran, versus su casi inexistencia antes de la vigencia de la LAM.

Estos datos evidencian, al menos cuantitativamente, que la LAM ha tenido un efecto muy importante en la cultura jurídica del país, acompañado de una persistente campaña de difusión. Por explicarlo de algún modo, la intención inicial de la implementación de LAM fue introducir en el pensamiento de los abogados, especialmente de aquellos que circulan en el ámbito empresarial, la idea de que los conflictos podían solucionarse sin tener que acudir a la justicia estatal y que podían ser resueltos por personas que, de cierta manera, podían tener conocimientos mucho más especializados que los jueces ordinarios. Estos objetivos, sin duda se han logrado.

La LAM constituyó, en su momento, una normativa de avanzada en materia procesal en el Ecuador, que ha sido aceptada por 
Implementación (parcial) en Ecuador de principios de la Ley Modelo CNUDMI, sobre arbitraje comercial. Retrospectiva histórica y necesidades

la comunidad jurídica ecuatoriana satisfactoriamente. Ahora bien, todo producto debe ser juzgado de acuerdo al tiempo. En ese orden, la LAM despertó el interés de los operadores jurídicos por un sistema hasta ese momento prácticamente no nato, más allá de que contiene principios fundamentales del derecho moderno de arbitraje que ha permitido que en el Ecuador se vaya institucionalizando satisfactoriamente este sistema. Sin embargo, en Ecuador, aún existen vacíos que no es posible desconocerlos y que continúan poniendo trabas a la evolución de este sistema.

\subsection{Vacíos de la LAM}

Es indiscutible el aporte que ha representado la implementación de la LAM en la cultura jurídica ecuatoriana. Sin embargo, presenta algunos vacíos que se discutirán a continuación.

\subsubsection{Motivos de recusación de árbitros}

La LAM carece de principios generales sobre las causas que podrían provocar la recusación de árbitros. Así, el artículo 21 se limita a mencionar que "son causas de recusación de los árbitros las previstas en el Código de Procedimiento Civil". Esta disposición representa un retroceso para alcanzar los objetivos que discutimos previamente sobre (i) la exclusión de las normas procesales diseñadas para la justicia ordinaria, y (ii) la adopción de las mejores prácticas internacionales en materia de arbitraje.

La LAM, a diferencia de la Ley Modelo, no contiene regulación alguna sobre el deber de revelación de los árbitros, ni mucho menos los estándares que podrían generar dudas sobre su independencia e imparcialidad para conocer una controversia. Además, el otro problema grave es la referencia innecesaria al Código de Procedimiento Civil, que contiene motivos de recusación pensados para quienes actúan, permanentemente, como jueces. Las normas del Código de Procedimiento Civil o del actual Código General de Procesos no han sido diseñadas para ser aplicadas en 
el arbitraje y mucho menos se han creado considerando las particularidades del sistema arbitral, como, por ejemplo, que las partes pueden designar árbitros, que en muchas ocasiones los árbitros son abogados que ejercen su profesión, entre otros.

Por estos motivos, consideramos que la referencia de la LAM al Código de Procedimiento Civil en materia de recusación de árbitros es inapropiada. Sería mucho más acertado considerar la aplicación de instrumentos internacionales como las Reglas de la IBA sobre conflictos de intereses en Arbitraje Internacional ${ }^{46}$, que contienen directrices estudiadas y diseñadas precisamente para el sistema arbitral, pues entienden los fundamentos de este sistema y los fenómenos reales que giran en torno de él.

\subsubsection{Práctica de la prueba}

Uno de los desafíos más importantes que enfrenta el arbitraje comercial en el Ecuador es la falta de regulación sobre la práctica de la prueba. Además de las regulaciones que existen sobre la presentación de la prueba junto con la demanda y la contestación a la demanda, el único artículo que tiene relación con la práctica de la prueba en la LAM es su artículo 23, el cual prescribe que "[s]i antes de la expedición del laudo, el tribunal o las partes estiman que se necesitan otras pruebas o cualquier otra diligencia para el esclarecimiento de los hechos, de oficio o a petición de parte podrá ordenar que se practiquen señalando día y hora". Si bien la norma descrita es importante porque permite al tribunal arbitral o a las partes solicitar pruebas para mejor proveer en cualquier momento del arbitraje, esta es insuficiente o insatisfactoria para establecer un verdadero régimen de práctica general de la prueba.

El vacío de la LAM en esta materia ha provocado que en la práctica se recurra a las normas de procedimiento civil como normas supletorias de la LAM. Sin embargo, este comportamiento es inaceptable y contrario a los verdaderos fines del arbitraje. En

46. IBA, Directrices sobre conflictos de Intereses en Arbitraje Internacional, 2014. 
Implementación (parcial) en Ecuador de principios de la Ley Modelo CNUDMI, sobre arbitraje comercial. Retrospectiva histórica y necesidades

efecto, cuando los contratantes consideran la inclusión de un convenio arbitral para resolver sus pleitos, precisamente lo hacen para excluir la intervención de la justicia ordinaria y sus prácticas formalistas.

\subsubsection{Proceso de nulidad de laudos}

La LAM regula en su artículo 31 el proceso de nulidad que podrá solicitarse en contra de un laudo ${ }^{47}$. Esta disposición es interesante y se encuentra acorde a la Ley Modelo, pues aporta ciertas ventajas inherentes del arbitraje al sistema procesal ecuatoriano. Por ejemplo, establece que la acción de nulidad es el único mecanismo (ordinario) mediante el cual se puede impugnar un laudo arbitral. A pesar de que esta norma ha constituido un avance significativo en materia de arbitraje, existen aún vacíos sobre el procedimiento a seguirse para sustanciarla. Esto ha provocado incansables discusiones en el foro ecuatoriano e incluso resoluciones contradictorias sobre el procedimiento que ha de seguirse $^{48}$.

47. LAM, N. 19, Art. 31 "Cualquiera de las partes podrá intentar la acción de nulidad de un laudo arbitral, cuando: a) No se haya citado legalmente con la demanda y el juicio se ha seguido y terminado en rebeldía. Será preciso que la falta de citación haya impedido que el demandado deduzca sus excepciones o haga valer sus derechos y, además, que el demandado reclame por tal omisión al tiempo de intervenir en la controversia; b) No se haya notificado a una de las partes con las providencias del tribunal y este hecho impida o limite el derecho de defensa de la parte; c) Cuando no se hubiere convocado, no se hubiere notificado la convocatoria, o luego de convocada no se hubiere practicado las pruebas, a pesar de la existencia de hechos que deban justificarse; d) El laudo se refiera a cuestiones no sometidas al arbitraje o conceda más allá de lo reclamado; o, e) Cuando se hayan violado los procedimientos previstos por esta Ley o por las partes para designar árbitros o constituir el tribunal arbitral. Del laudo arbitral podrá interponerse ante el árbitro o tribunal arbitral, acción de nulidad para ante el respectivo presidente de la corte superior de justicia, en el término de diez días contado desde la fecha que éste se ejecutorió. Presentada la acción de nulidad, el árbitro o tribunal arbitral dentro del término de tres días, remitirán el proceso al presidente de la corte superior de justicia, quien resolverá la acción de nulidad dentro del término de treinta días contados desde la fecha que avocó conocimiento de la causa. La acción de nulidad presentada fuera del término señalado, se tendrá por no interpuesta y no se la aceptará a trámite. Quien interponga la acción de nulidad, podrá solicitar al árbitro o tribunal arbitral que se suspenda la ejecución del laudo, rindiendo caución suficiente sobre los perjuicios estimados que la demora en la ejecución del laudo pueda causar a la otra parte. El árbitro o tribunal arbitral, en el término de tres días, deberán fijar el monto de la caución, disponiendo la suspensión de la ejecución del laudo. La caución deberá constituirse dentro del término de tres días, contados a partir de esta notificación".

48. Para observar una mayor discusión sobre este tema, véase, E. CARMigniani, H. García y C. CEPEDA, "Arbitraje en el Ecuador. Desarrollo Jurisprudencias y Reformas Legales Recientes", Revista Ecuatoriana de Arbitraje, No. 7, 2015, «http://iea.ec/pdfs/2015/Art6.pdf». 
En definitiva, la forma en la cual debe tramitarse la acción de nulidad en el Ecuador es un área gris en el arbitraje ecuatoriano. Hasta la fecha no existe armonía sobre el proceso y recursos que pueden presentarse. Las resoluciones dispares y contradictorias de los más altos tribunales han creado solo confusión y caos.

\subsection{Desafíos que enfrenta el arbitraje ecuatoriano}

Es muy claro que la LAM no es perfecta y que, por el contrario, tiene una serie de vacíos e inconsistencias con los objetivos del arbitraje como método de resolución de disputas. Solucionarlos es parte de los desafíos que enfrenta el arbitraje en el Ecuador y quizá no es el más importante.

Hoy en día, el arbitraje es una institución aceptada y que funciona en forma relativamente eficiente. Sin embargo, el problema principal sigue siendo cultural. En palabras de César Coronel Jones, el arbitraje ha enfrentado

[...] dificultades, resistencias y temores, especialmente por parte de los operadores jurídicos: abogados, jueces e inclusive los propios árbitros quienes, formados con las ideas de respeto profundo a las normas procesales e inclusive inclinados hacia el formalismo y ritualismo en la práctica litigiosa, se ven abocados a lidiar con una modalidad de resolución de controversias que privilegia ampliamente el contenido sobre la forma, la agilidad y celeridad por sobre el rito y las fórmulas sacramentales ${ }^{49}$.

Desde nuestra perspectiva, el principal problema que enfrenta el arbitraje comercial en Ecuador es la idea que mantienen árbitros y abogados de que el arbitraje es una especie de calco del proceso civil, únicamente con cambio de jueces, y que, en consecuencia, especialmente, en temas probatorios hay que seguir los ritualismos y procedimentalismos a ultranza previstos en las normas procesales. Esto es lo que impide que el sistema continúe su evolución.

49. C. Coronel Jones, “Arbitraje y Procedimiento”, Iuris Dictio, No. 11, 2007, p. 37. 
Implementación (parcial) en Ecuador de principios de la Ley Modelo CNUDMI, sobre arbitraje comercial. Retrospectiva histórica y necesidades

A este problema lo acompaña el desconocimiento o falta de comprensión de los abogados e incluso de los árbitros de que, a la luz del ordenamiento jurídico ecuatoriano, la naturaleza jurídica del arbitraje es esencialmente negocial. Esto otorga flexibilidad a las partes y también al tribunal arbitral para diseñar el proceso más idóneo para resolver una disputa concreta. Es sustancial que los operadores del sistema arbitral comprendan que el arbitraje es un proceso flexible, porque permite a las partes y al tribunal arbitral adoptar las mejores prácticas, las normas más convenientes y eficientes para resolver su controversia. Así, por oposición al orden planificado del proceso civil, el proceso arbitral es autónomo y, para preservar esta naturaleza, en principio, las normas procedimentales de los juicios ordinarios no le son aplicables ${ }^{50}$.

\section{CONCLUSIÓN; MODERNIZACIÓN DEL ARBITRAJE ECUATORIANO}

La Ley Modelo es uno de los instrumentos más importantes a nivel internacional en materia de arbitraje. Sus objetivos principales son unificar las prácticas arbitrales a nivel mundial y garantizar que este mecanismo de resolución de disputas funcione de forma efectiva. En el Ecuador, se han implementado varios de los principios y lineamientos de la Ley Modelo en la LAM, normativa que se convirtió en el instrumento esencial para implementar la figura del arbitraje en el Ecuador.

Veinte años después de la promulgación de la LAM, nos encontramos con que el arbitraje comercial es un mecanismo de resolución de controversias que ya funciona en el Ecuador. Esto es satisfactorio, pues es indiscutible el crecimiento y difusión que ha tenido en los últimos tiempos. Sin embargo, es indudable que hay complicaciones y desafíos que el arbitraje aún debe enfrentar, como los vacíos legislativos en la materia y la falta de comprensión del sistema por parte de sus usuarios.

50. A. Galindo y H . García, "Relación entre el Código Orgánico General de Procesos y el procedimiento arbitral”, Revista Ecuatoriana de Arbitraje No. 6, 2014, p. 56. 
Esto nos lleva a la necesidad de continuar con lo que hemos denominado la segunda fase del arbitraje en el Ecuador, de modernización del sistema. En esta nueva época del arbitraje ecuatoriano es fundamental superar las trabas que se han enunciado en este artículo y para ello hay soluciones como: (i) la celebración de convenios arbitrales más "sofisticados", en los que por ejemplo se acuerde la aplicación de ciertas reglas procedimentales o se acuerde la aplicación de directrices modernas, por ejemplo, las reglas IBA para la práctica de la prueba y para conflictos de intereses en arbitraje; (ii) la modernización de reglamentos de arbitraje de las instituciones encargadas de administrar estos procesos, considerando la experiencia que han podido adquirir en estos veinte años; (iii) finalmente, se podría pensar en una reforma legislativa que complemente las disposiciones de la LAM para brindar claridad y mayor fuerza al sistema, sobre todo en esas áreas que continúan siendo grises como la práctica de la prueba y el proceso de nulidad de laudos arbitrales.

En definitiva, la implementación de la Ley Modelo en el Ecuador a través de la LAM fue un importante paso. Ahora, el reto consiste en superar los desafíos culturales y legislativos que el arbitraje en el Ecuador aún enfrenta. 


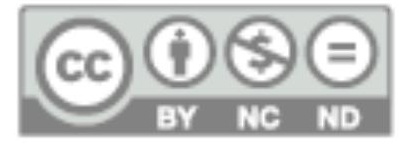

